\title{
A rare case of sigmoid colon duplication in an adult man
}

\author{
Subramaniyam Raviraj, Ahilan Nadesan \\ Teaching Hospital, Jaffna, Sri Lanka
}

Key words: Colonic duplication; adult man; constipation; abdominal pain

\section{Introduction}

Intestinal duplication is a rare congenital abnormality with the incidence rate of 1 in 4000-5000 births. The ileum is the most common site for intestinal duplication. The Colonic duplications are the rarest condition among Intestinal duplications. It representing less than $10-15 \%$ of all intestinal duplications [2]. The colonic duplication in an adult is an extremely rare condition. It commonly occurs in children and may proceed unrecognized until adulthood as in this patient. The preoperative diagnosis was uncertain as in this patient and the definitive diagnosis was only made during the laparotomy and later confirmed with the histopathology report.

\section{Case presentation}

A 34 years old man presented to the surgical clinic with a history of right sided abdominal pain for 3 months duration. He had abdominal colic pain in the right side abdomen and these episodes usually associated with decreased bowel opening for 4-5 days then subsequently relieved by passage of hard stools. The pain was severe and colicky. During this period he noted mild distension of lower abdomen. He denied the history of diarrhoea with blood or mucus in the past. $\mathrm{He}$ underwent a lower midline laparotomy at the age of 10 years for recurrent abdominal pain. The patient was not aware of the details regarding this laparotomy and also didn't have any documents regarding this past illness. Even after this surgery, he continued to have similar symptoms with the same intensity.

Examination revealed a non-distended and mildly tender abdomen with lower midline scar. Abdominal examination and a digital rectal examination were within the normal limits. Other systemic examinations were found to be normal.

His blood investigation including $\mathrm{WBC}, \mathrm{Hb}$, renal function tests and liver function tests were within the normal range.

\footnotetext{
Correspondence: N.Ahilan

E-mail: ahilann@gmail.com

Received: 07-03-2019 Accepted: 11-06-2019

(iD) http://orcid.org/0000-0002-8069-2240

DOI: http://doi.org/10.4038/sljs.v37i2.8630
}

Plain abdomen X-ray was inconclusive. Ultrasonic examination of Abdomen and pelvis revealed a segment of dilated bowel loop without any other significant findings. He underwent for Contrast-Enhanced Computed Tomography of abdomen and pelvis which revealed a dilated small bowel loops seen probably in an ileal area with a collapsed rectum and large bowel. The conclusion made by consultant radiologist was highly suspicious of chronic intussusception.

An exploratory laparotomy was carried out and there was a distended blind bowel loop with impacted faeces found to be extending from the sigmoid colon to the right side of the abdomen retroperitoneally. This loop was large enough to cross the midline and extending towards the right side of the abdomen retroperitoneally. There was no thickened fibrotic area was noted between the blind loop and the sigmoid junction to suggest a previous anastomosis. This blind bowel loop was mobilized from other retroperitoneal structures and it was resected along with the part of the sigmoid colon from which the loop was arising. Y shaped loop was resected out and end to end anastomosis was carried out between the two ends of the sigmoid colon.

\section{Discussion}

Alimentary tract duplication is a very rare congenital abnormality. The small intestine is the most common region in the gastrointestinal tract which will be affected by Intestinal Duplication. Colorectal duplication is the least common type among gastrointestinal duplication. A wide spectrum of clinical presentation and radiological features of colonic duplication is creating diagnostic difficulties for surgeons and radiologist [1].

The symptoms of colonic duplication are usually nonspecific and also depend on the type of duplication and also depend on the associated abnormalities [3]. This congenital condition remains frequently silent for several years if there is no complication. The few of the presentations of colonic duplications are intra-abdominal mass, chronic abdominal pain [as in this patient], constipation [as in this patient] and intestinal obstruction due to the impact of faeces or compressing adjacent bowel by faecal loaded distended duplication of the colon. Rarely these patients can present with volvulus, intussusception, bleeding or perforation. 
If there is no associated malformation in a patient with colonic duplication, most of them remain silent for several years until they developed a complication. The real challenge for the surgeon is the clinical and radiological diagnosis before the definite treatment. It's more applicable in the case of Y shape colonic duplication as in this patient [1]

The CT or MRI after Barium enema administration demonstrating the additional intestinal loop arising from the native colon or the bifurcation of colonic lumen detected during colonoscopy examination will help to diagnose the colonic duplication before definitive treatment. The radiological diagnosis was made as highly suspicious of chronic intussusception of the small intestine in this patient. We didn't proceed with further radiological investigations such as MRI or CEST and decided for laparotomy.

The possibility of a bowel anastomosis and a blind loop left inside during the previous childhood surgery is unlikely since there was no intraoperative evidence of previous scarring or thickened area in the Junction of Y loop and histological features of biopsy specimen suggesting of colonic duplication.

Once the diagnosis is confirmed or suspected, the recommended management plan is surgical resection of the

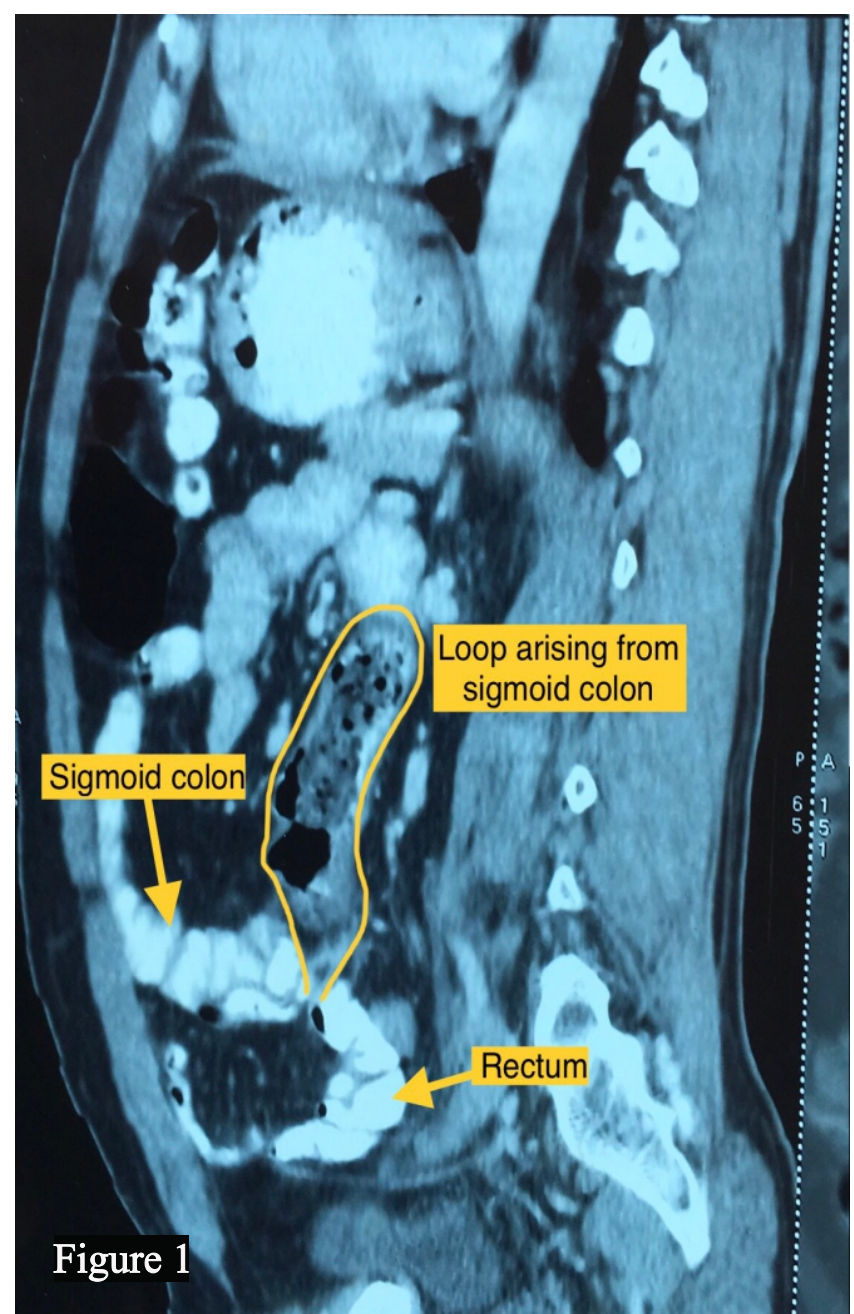

duplication together with the attached normal colonic segment. The complete resection of duplication with the attached normal colon will prevent the minor potential risk of future malignancy, the effect of ectopic gastric mucosa and mucoviscidosis [1], [2].

\section{Conclusion}

We conclude the blind loop arising from the sigmoid colon is a sigmoid colonic duplication. The vague presentation, previous laparotomy and Radiological Suspicious of intussusception of small intestine made us difficulty in marking the definite diagnosis before Surgery.

The suspicious of colonic duplication should be borne in mind When treating an adult patient with chronic abdominal and constipation even though it's a rare condition. The CT and MRI combined with barium enema may solve the diagnostic dilemma before Surgery.

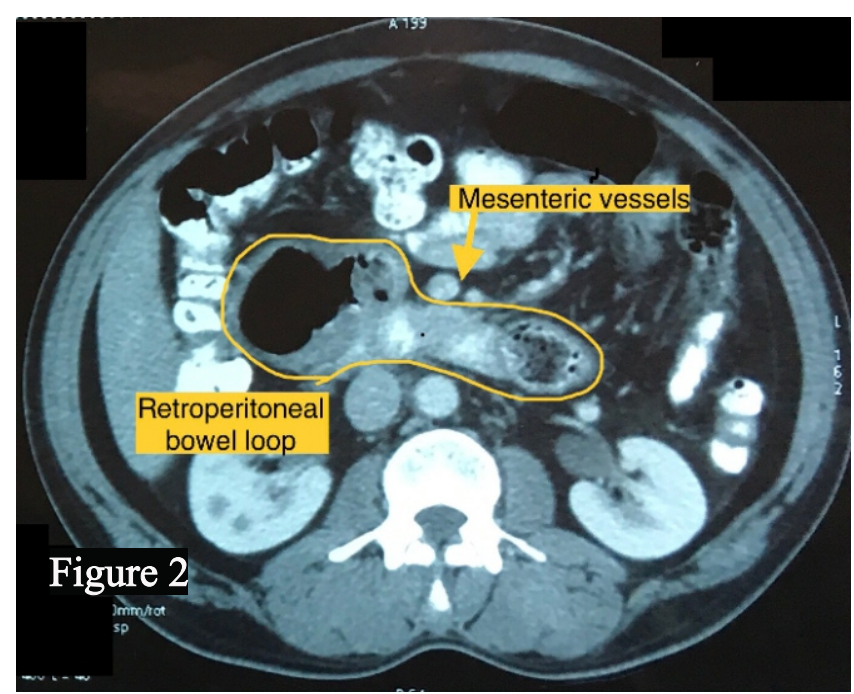

Figure 1 and 2. CT images show the bowel loop arising form sigmoid colon

All authors disclose no conflict of interest. The study was conducted in accordance with the ethical standards of the relevant institutional or national ethics committee and the Helsinki Declaration of 1975, as revised in 2000 .

\section{References}

1. Al-Jaroof, A. H., Al-Zayer, F., \& Meshikhes, A.-W. N. [2014]. A case of sigmoid colon duplication in an adult woman. BMJ Case Reports, 2014. https://doi.org/10.1136/bcr-2014-203874

2. Chang, H.-C., Huang, S.-C., Chen, T.-C., Lai, M.-W., Chen, S.-Y., \& Lai, J.-Y. [2011]. Y-Shaped Colonic Duplication: Report of A Case and Literature Review. Chang Gung Med J [Vol. 34]. Retrieved from https://pdfs.semanticscholar.org/1f57/1cdf7cdc90d606131b0a9 8c12b09b35dd87f.pdf

3. Banchini, F., Delfanti, R., Begnini, E., Tripodi, M. C., \& Capelli, P. [2013]. Duplication of the transverse colon in an adult: case report and review. World Journal of Gastroenter-ology, 19[4], 586-589. https://doi.org/10.3748/wjg.v19.i4.586 


\section{Learning Points:}

- Colonic duplication in an adult is an extremely rare condition.

- The presentation of colonic duplication is usually nonspecific.

- MRI, CT colonoscopy combined with barium enema will be considered in these patients to achieve a definite diagnosis 